Background In low-risk women, in vitro inhibition of E. coli by genital tract secretions is associated with Lactobacillus crispatus and jensenii proteins. However in at-risk populations, HIV seroconversion was associated with greater E. coli bactericidal activity and inflammatory immune mediators. We therefore analysed the relationship between inflammation, E. coli bactericidal activity, and microbiota in vaginal swabs from participants in a safety study of $\mathrm{VivaGel}^{\oplus}$

Methods Swabs were collected before and after product use from subjects randomised to vaginal $\mathrm{VivaGel}{ }^{\circledR}(\mathrm{n}=66)$, VivaGel ${ }^{\circledR}$ placebo $(n=65)$, or hydroxyethylcellulose (HEC) placebo $(n=54)$. Cytokines were quantified by multiplex proteome array and lactoferrin and SLPI by ELISA to generate a cumulative inflammatory score using principal components analysis. E. coli bactericidal activity in swab supernatants was quantified by a colony reduction assay. Vaginal bacteria were characterised by quantitative cultures. Generalized estimating equations controlling for product use were used for analyses.

Results Higher inflammatory score was associated with detection of Gardnerella vaginalis (OR 1.5; $p=0.02)$ and anaerobic grammenegative rods (OR 1.4, $\mathrm{p}=0.03$ ), a trend towards diminished hydrogen peroxide-producing lactobacilli (OR 0.7, $p=0.1$ ), and increased E. coli bactericidal activity $(p<0.001)$. The combined presence of group B streptococcus, E. coli, S. aureus, and enterococcus (potential pathogens) was associated with decreased E. coli bactericidal activity $(p=0.06)$. However these results were modified by gel type. Higher inflammatory score was associated with greater E. coli bactericidal activity only in the placebo arms $\left(\operatorname{VivaCel}^{\circledR} \mathrm{p}<0.001\right.$; HEC $p=0.002$, while pathogenic bacteria were associated with decreased E. coli bactericidal activity in the VivaGel ${ }^{\circledast}$ arm $(p=0.001)$.

Conclusion Mucosal inflammation was associated with E. coli bactericidal activity in women using placebo gels, which could contribute to the previously observed link between bactericidal activity and HIV seroconversion. However bactericidal activity in women using VivaGel ${ }^{\circledR}$ was influenced by pathogenic bacterial populations, which may reflect an altered genital mucosal milieu.

\section{P3.364 DOES TENOFOVIR GEL - OR DO OTHER VAGINAL MICROBICIDE PRODUCTS - AFFECT DETECTION OF BIOMARKERS OF SEMEN EXPOSURE?}

doi:10.1136/sextrans-2013-051184.0817

'M C Snead, 'A P Kourtis, '2J H Melendez, 'C M Black, ${ }^{3} \mathrm{C}$ K Mauck, ${ }^{2} \mathrm{D}$ M Chaney, ${ }^{3} \mathrm{G}$ Doncel. ' $\mathrm{CDC}$, Atlanta, GA, United States; 'JHU, Baltimore, MD, United States; ${ }^{3}$ CONRAD, Eastern Virginia Medical School (EVMS), Arlington, VA, United States

Background Prostate Specific Antigen (PSA) and Y chromosome are used as biomarkers of semen exposure. There is currently no information on whether vaginal products evaluated in microbicide trials, in particular, tenofovir gel (TFV), UC781 or the universal HEC placebo (hydroxyethylcellulose) affect the detection of these markers.

Methods Dilutions of TFV, UC781, and the universal HEC placebo, combined with semen, were tested for PSA using both the Abacus ABAcard semi-quantitative and the Abbott Architect quantitative assay. Y chromosome DNA was determined by real-time polymerase chain reaction (real-time PCR).

Results Tenofovir gel and the universal HEC placebo adversely affected PSA detection using the ABAcard, but not the Abbott Architect PSA assay. UC781 affected PSA results by ABAcard and, at all dilutions, caused invalid results for Abbott Architect assay. None of the products substantially affected the $\mathrm{Y}$ chromosome PCR assay's indication of presence of Y chromosome DNA, but the quantitative results varied by product.

Conclusions These in vitro results indicate that the Abbott Architect assay, rather than the ABAcard, should be used for PSA detection when tenofovir gel or the universal HEC placebo might be present. The Y chromosome PCR assay when used qualitatively to indicate presence of $Y$ chromosome is not affected by any of these products, but the quantitative results should be used cautiously as the values are affected by microbicide products. In vivo confirmation of these findings is recommended to further optimise detection of semen biomarkers when microbicide products may be used.

\section{P3.365 TENOFOVIR VAGINAL FILIM: SAFETY ASSESSMENT IN THE MACAQUE MODEL}

doi:10.1136/sextrans-2013-051184.0818

'D L Patton, 'Y Cosgrove Sweeney, ${ }^{2} \mathrm{~L} C$ Rohan, ${ }^{2} \mathrm{~S}$ L Hillier. 'U of WA, Seattle, WA, United States; ' 2 Magee Womens Research Institute, Pittsburgh, PA, United States

Background Tenofovir, a nucleoside reverse transcriptase inhibitor (NRTI), has been formulated as a vaginal film for topical microbicide development. To help inform studies to be conducted in a human population, we assessed safety factors in the pigtailed macaque model.

Methods The use of monkeys on this protocol was approved by the Institutional Animal Care and Use Committee at the University of Washington. Animals were handled humanely, and experiments were performed within the National Institutes of Health's animal use guidelines, in compliance with the Guide for the Care and Use of Laboratory Animals and the Animal Welfare Act. Six pigtailed macaques underwent a three-arm cross-over design protocol comparing two doses of tenofovir films (40mg; $20 \mathrm{mg}$ ) to a placebo film. Films used were $1 / 3$ the size intended for human use. The drug content of macaque-sized test films was $11.2 \pm 0.4$ and $5.1 \pm 0.3 \mathrm{mg}$ tenofovir/ film. We administered the vaginal films to pigtailed macaques daily for five days one week, followed by four days the next week. Safety of repeated, daily exposures was measured by repeated colposcopic assessment, vaginal $\mathrm{pH}$, vaginal smear and microbiology tracking.

Results Colposcopy revealed similar tissue appearance in all three study arms. There were no indications of product induced tissue disruption to vaginal or cervical mucosal surfaces. Vaginal microbiology assessments revealed similar shifts in flora prevalence across both the tenofovir formulations and the placebo study arm. Vaginal $\mathrm{pH}$ fluctuated similarly across all three study arms. Polymorphonuclear cell counts determined from Gram stained vaginal smears increased somewhat with exposure to the higher dose tenofovir formulation compared to the lower dose tenofovir and the placebo arms.

Conclusions Quick dissolving vaginal films, formulated to deliver tenofovir as a topical microbicide, are a promising alternative to gel formulations. Well designed clinical assessments of tenofovir film safety, pharmacokinetics, pharmacodynamics and product dispersal are warranted and ongoing.

\section{P3.366 CHLAMYDIA SCREENING IN EDUCATIONAL SETTINGS: A SYSTEMATIC REVIEW OF STRATEGIES AND OUTCOMES}

doi:10.1136/sextrans-2013-051184.0819

'M S Jamil, ${ }^{2 H}$ M Bauer, 'H Ali, '3 S Hocking, ${ }^{4} \mathrm{~J}$ Walker, 1,5B Donovan, ' $\mathrm{J}$ Kaldor, 'R Guy. 'The Kirby Institute, University of New South UK, Sydney, Australia; 'Division of Epidemiology, School of Public Health, University of California, Berkeley, CA, United States; ${ }^{3}$ Center for Women's Health, Gender and Society, Melbourne School of Population Health, University of Melbourne, Melbourne, Australia; ${ }^{4}$ Centre for Excellence in Rural Sexual Health, Rural Health Academic Centre, Melbourne Medical School, University of Melbourne, Melbourne, Australia; ${ }^{5}$ Sydney Sexual Health Centre, Sydney Hospital, Sydney, Australia

Background Low Chlamydia trachomatis (CT) testing rates in primary-care (5-20\%) in many countries have encouraged screening programmes in non-clinical settings. We describe the strategies and outcomes of screening programmes in educational settings. 